\title{
Maternal Serum Cancer Antigen 125: A Marker of Severity of Preeclampsia
}

\author{
Geya Gottipati ${ }^{1}$, Kantharaju Supriya ${ }^{2}$
}

\begin{abstract}
Aim: To study the levels of cancer antigen 125 (CA-125) in normal, preeclampsia, and gestational hypertension study groups and correlate with other parameters which mark the severity of the disease.

Materials and methods: A cross-sectional study was conducted at a Tertiary Care Hospital, Mangaluru, India. A total of 165 subjects were divided into three study groups of preeclampsia, gestational hypertension, and normal pregnancy, and each group consisted of 55 subjects. Cancer antigen 125 levels were measured in these study groups.

Results: Mean value of CA-125 value in the preeclampsia group was $56.7 \mathrm{IU} / \mathrm{mL}$ which was significantly higher compared to other two study groups. Cancer antigen 125 was found to have a positive correlation with systolic blood pressure ( $r$ value $=0.4, p$ value $=0.001$ ), diastolic blood pressure $(r$ value $=0.3, p$ value $=0.001)$, uric acid $(r$ value $=0.2, p$ value $=0.001)$, and also an association was observed between increasing CA-125 and proteinuria ( $p$ value $=0.001$ ) oligohydramnios ( $p$ value $=0.001$ ) fetal growth restriction ( $p$ value $=0.03$ ) and onset of the disease ( $p$ value $=0.001)$. However, a negative correlation with platelets $(r$ value $=-0.14, p$ value $=0.06)$ and birth weight $(r$ value $=-0.113, p$ value $=$ $0.15)$ was observed.

Conclusion: Cancer antigen 125 is a marker indicating increasing severity of the disease. It is a biochemical marker which can be used in screening of preeclampsia. Further studies are needed for the evaluation of raising serial CA-125 levels as a marker of progression of disease.

Clinical significance: Hypertensive disorders of pregnancy are one of the most common causes of death due to pregnancy. Cancer antigen 125 was proposed to be elevated in patients with hypertension due to chronic inflammation. Hence CA-125 levels if used as screening tool can estimate the severity of the disease and help clinicians in identifying women who are at risk for further complications and act accordingly. Keywords: CA-125 levels, Gestational hypertension, Preeclampsia.

Journal of South Asian Federation of Obstetrics and Gynaecology (2019): 10.5005/jp-journals-10006-1671
\end{abstract}

\section{INTRODUCTION}

Hypertensive disorders of pregnancy are one of the most common causes of death due to pregnancy. ${ }^{1}$ Incidence of preeclampsia alone is identified in $3.9 \%$ of all pregnancies. ${ }^{2}$ Cancer antigen 125 (CA-125) or MUCIN 16 is a membrane protein and possesses a single transmembrane domain and it is the most frequently used biomarker in ovarian cancer detection. ${ }^{3}$ Decidual cells affected by chorionic invasion or placental separation are a source of CA-125. Underlying mechanism proposed for the increased CA-125 levels in preeclampsia is an extension of decidual destruction and separation of trophoblasts from the decidua. ${ }^{4}$ This theory has been supported by various studies and they have proposed that increasing levels of CA-125 is an indicator of increasing severity of the disease. All the studies so far compared levels of CA-125 in mild and severe preeclampsia with that of normal pregnancy. In the present study, we compared levels of CA-125 in gestational hypertension and preeclampsia with that of normal pregnancy. Relation of CA-125 levels with various other outcomes and parameters of preeclampsia were analyzed, and a mean value of CA-125 in the gestational hypertension and preeclampsia groups has been derived.

\section{Materials and Methods}

This study was a cross-sectional study performed during a study period of 2 years from September 2014 to July 2016 in a Tertiary Care Center after obtaining the Institutional Ethics Committee approval. A total of 165 subjects divided into three study groups between 20- and 42-week period of gestation with singleton pregnancies fulfilling inclusion criteria were included. Written informed verbal
1,2Department of Obstetrics and Gynaecology, Kasturba Medical College, Manipal University, Mangaluru, Karnataka, India

Corresponding Author: Geya Gottipati, Department of Obstetrics and Gynaecology, Kasturba Medical College, Manipal University, Mangaluru, Karnataka, India, Phone: +91 97333790529, e-mail: geya. gottipati@gmail.com

How to cite this article: Gottipati G, Supriya K. Maternal Serum Cancer Antigen 125: A Marker of Severity of Preeclampsia. J South Asian Feder Obst Gynae 2019;11(3):153-155.

Source of support: Nil

Conflict of interest: None

consent was obtained from all the subjects. Three study groups included normal pregnancy group, gestational hypertension group, and preeclampsia group consisting of 55 subjects each. Preeclampsia was diagnosed and classified according to the criteria specified by the technical bulletin of the American College of Obstetricians and Gynecologists and the National High Blood Pressure Education Program Working Group Report on High Blood Pressure in Pregnancy. Multiple gestation and patients with chronic hypertension, ovarian diseases, tuberculosis, overt diabetes mellitus, and renal disease were excluded. Blood pressure recording was done in the right arm sitting position venous blood samples for complete blood count, renal function test, uric acid, liver function test, and the CA-125 levels were drawn from all participants. Routine urine analysis was done in all participants and 24 hours urine protein was done. Samples collected were centrifuged within 2 hours of collection and the serum was stored 
at -20 degree Celsius. Samples collected were processed within 3 months of collection. An enzyme linked immunosorbent assay (ELISA) was used to estimate the CA-125 levels.

\section{Statistical Analysis}

Data were analyzed using Chi-square test, Fisher's exact test, and Pearson's correlation coefficient. $p$ value $<0.05$ was considered significant, $r<0.5$ is weak correlation, $>0.5$ mild strength, and $>0.7$ strong correlation. The $r+$ is direct correlation and $r-$ is inverse correlation. A statistical package SPSS (Chicago, Illinois) version 17.0 was used for data analysis.

\section{Results}

All three study groups were comparable in demographic characters such as age, parity, and period of gestation. Preeclampsia had a mean systolic BP of $153.6 \mathrm{~mm} \mathrm{Hg}$ and diastolic BP of $101.9 \mathrm{~mm} \mathrm{Hg}$ which was statistically highly significant ( $p$ value $=0.000$ ). Mean systolic BP in gestational hypertension was $141.8 \mathrm{~mm} \mathrm{Hg}$ and diastolic BP was $91.6 \mathrm{~mm} \mathrm{Hg}$. Mean birth weight among study subjects in preeclampsia is $2.1 \mathrm{~kg}$ which is lower compared to other two study groups ( $p$ value $=0.000$ ). Mean gestational age at the onset of disease is 31 weeks for the preeclampsia group and 35 weeks for the gestational hypertension group indicating early onset of disease in the preeclampsia group ( $p$ value $=0.000$ ).

Table 1 shows mean value of CA-125 in the preeclampsia group was $56.6 \mathrm{IU} / \mathrm{mL}$ which was highly significant compared to other two study groups $(p=0.000)$.

Table 2 shows CA- 125 had a significant association with the onset of the disease $(p=0.000)$ indicating that earlier the onset of disease higher the value and had significant association with other markers of severity of disease such as uric acid $(p=0.000)$, proteinuria $(p=0.000)$, fetal growth restriction $(p=0.000)$, and oligohydramnios $(p=0.000)$.

As shown in Table 3, CA-125 had positive correlation with systolic BP $(r=0.4)$, diastolic BP $(r=0.3)$, uric acid $(r=0.2)$, creatinine $(r=0.2)$, and body mass index (BMI) $(r=0.1)$, and negative correlation with birth weight $(r=-0.113)$ and platelets $(r=-0.145)$.

Cutoff value of CA-125 in the present study is $23.7 \mathrm{IU} / \mathrm{mL}$ with a sensitivity and specificity of $83.6 \%$ and $98.2 \%$ obtained from the receiver operating characteristic (ROC) curve as shown in Figure 1.

\section{Discussion}

Preeclampsia is a disease specific to pregnancy affecting almost every organ. ${ }^{5}$ Reduced trophoblastic migration into the maternal decidua in preeclampsia causes chronic inflammation leading to increased expression of CA-125. ${ }^{6}$

Table 4 depicts comparison of mean values and cutoff values of CA-125 with other studies. Mean value of CA-125 in the preeclampsia group in the present study was $56.6 \mathrm{IU} / \mathrm{mL}$ which was similar to Bhattacharya and $\mathrm{Saha}^{7}(58.5 \mathrm{IU} / \mathrm{mL})$, whereas value was slightly higher when compared to Karaman et al. ${ }^{8}(38.8 \mathrm{IU} / \mathrm{mL})$ and Hassan et al. ${ }^{9}(38.04 \mathrm{IU} / \mathrm{mL})$. This difference in mean can be explained by wide difference in the standard deviation obtained in the present study as much higher values of CA-125 were observed in a few patients with severe form of disease resulting in higher mean values compared to other two above-mentioned studies.

Cancer antigen 125 values of gestational hypertension group of the present study were comparable to the mild preeclampsia group of Karaman et al. ${ }^{8}(18.8 \mathrm{IU} / \mathrm{mL})$. Bhattacharya and Saha ${ }^{7}$ $(53.7 \mathrm{IU} / \mathrm{mL})$ and Hassan et al. ${ }^{9}(32.5 \mathrm{IU} / \mathrm{mL})$ observed much higher value of CA-125 in the mild preeclampsia group compared to the gestational hypertension group. This difference in results can be explained by increased inflammatory response in preeclampsia when compared to gestational hypertension leading to increased release of CA-125 which is proposed as an inflammatory marker. In the present study, difference between mean values of CA-125 in gestational hypertension and preeclampsia is noted. This is in favor of hypothesis that increasing severity of the disease leads to increased release of CA- 125 .

Cutoff value of CA-125 in the present study is $23.7 \mathrm{IU} / \mathrm{mL}$ with a sensitivity and specificity of $83.6 \%$ and $98.2 \%$ which is lower compared to other studies. Ozat et al. ${ }^{10}$ obtained a cutoff value of $50 \mathrm{IU} / \mathrm{mL}$ with a sensitivity of $93.7 \%$ and specificity of $88 \%$. But the specificity of cutoff value of the present study is higher. Cebesoy et al. ${ }^{6}$ derived a cutoff value of $35 \mathrm{IU} / \mathrm{mL}$ but not measured sensitivity and specificity. This difference in cutoff values can probably be due to the inclusion of severe preeclampsia as a separate group in other studies.

Cancer antigen 125 was found to have a positive correlation with systolic blood pressure, diastolic blood pressure, mean arterial pressure (MAP), uric acid, creatinine, and total urinary concentration, and negative correlation with platelets and birth weight in the present study.

Table 1: Distribution of CA-125 in IU/mL

\begin{tabular}{lcrll}
\hline CA-125 & Mean & \multicolumn{1}{c}{ SD } & \multicolumn{1}{l}{ Range } & pvalue \\
\hline CA-125-gestational hypertension & 15.7 & 8.4 & $13.4-17.9$ & $0.000(\mathrm{HS})$ \\
Preeclampsia & 56.6 & 88.2 & $32.7-80.4$ & \\
Normal pregnancy & 7.9 & 4.1 & $6.8-9.02$ & \\
\hline
\end{tabular}

HS, highly significant

Table 2: Association of CA-125 with other parameters

\begin{tabular}{llllll}
\hline & Onset & Uric acid & Proteinuria & FGR & Oligohydramnios \\
\hline$p$ value & 0.001 & 0.001 & 0.001 & 0.030 & 0.001 \\
\hline
\end{tabular}

FGR, fetal growth restriction

Table 3: Correlation of CA-125 with other parameters

\begin{tabular}{llllllll}
\hline & $S B P$ & $D B P$ & Platelet & Uric acid & Birth weight & BMI & Creatinine \\
\hline$r$ value & 0.4 & 0.3 & -0.145 & 0.2 & -0.113 & 0.1 & 0.2 \\
$p$ value & 0.000 & 0.000 & 0.06 & 0.000 & 0.15 & 0.18 & 0.003 \\
\hline
\end{tabular}

DBP, diastolic blood pressure; SBP, systolic blood pressure 


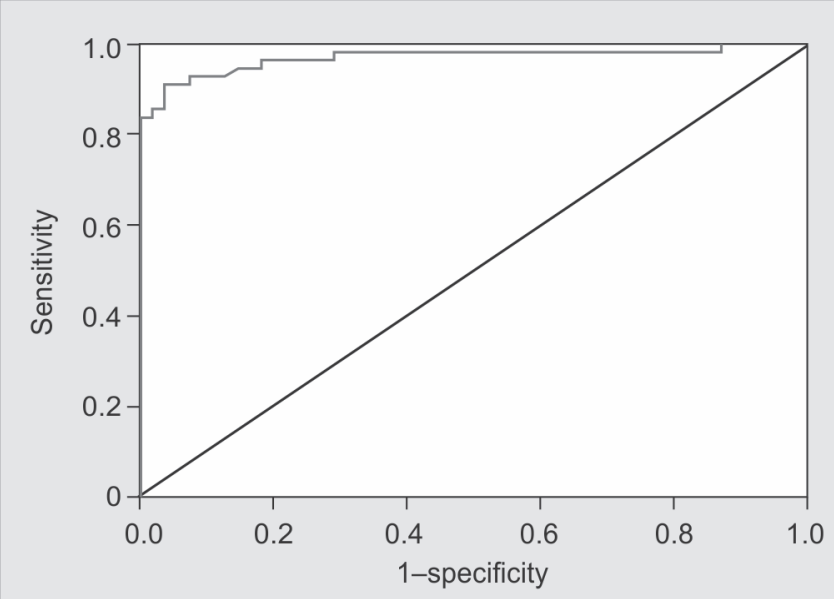

Fig. 1: Receiver operating characteristic (ROC) curve obtained for CA125 values

Table 4: Comparison of mean and cutoff values of CA-125 with other studies

\begin{tabular}{|c|c|c|c|}
\hline \multirow[b]{2}{*}{ Study } & \multicolumn{2}{|c|}{ Mean value (IU/mL) } & \multirow{2}{*}{$\begin{array}{l}\text { Cutoff value } \\
\text { (IU/mL) } \\
\text { Preeclampsia }\end{array}$} \\
\hline & $\begin{array}{l}\text { Mild preeclamsial } \\
\text { gestational } \\
\text { hypertension }\end{array}$ & $\begin{array}{l}\text { Severe } \\
\text { preeclampsia }\end{array}$ & \\
\hline $\begin{array}{l}\text { Bhattacharya and } \\
\text { Saha }^{7}\end{array}$ & 53.7 & 58.5 & 23.7 \\
\hline Karaman et al. ${ }^{8}$ & 18.8 & 38.8 & \\
\hline Hassan et al. ${ }^{9}$ & 32.5 & 38.04 & \\
\hline Ozat et al. ${ }^{10}$ & - & - & 50 \\
\hline Cebesoy et al. ${ }^{6}$ & - & - & 35 \\
\hline Present study & 15.7 & 56.6 & \\
\hline
\end{tabular}

Ozat et al. ${ }^{10}$ and Bhattacharya and Saha ${ }^{7}$ found a positive correlation between CA-125 and other variables similar to the present study. But platelets had a positive correlation with CA-125, whereas in the present study, platelets had a negative correlation. This difference in results can be explained due to variations in sample size.

Mean birth weight in the preeclampsia group in the present study is $2.1 \mathrm{~kg}$ indicating lower birth weight in the preeclampsia group probably due to fetal growth restriction which is one of the manifestations of the disease or preterm deliveries due to labor induction for termination of pregnancy.

Similar outcomes were found by Karaman et al., ${ }^{8}$ wherein the mean birthweight was lower in mild and severe preeclampsia group. Mean values of age, BMI, serum creatinine, and platelets are comparable to the present study.

In the study done by Bhattacharya and Saha, ${ }^{7}$ the estimated fetal weight in all three study groups is similar which differs from the present study probably due to the difference in inclusion criteria, as the study population included pregnant women beyond 32 weeks period of gestation only unlike the present study which included women beyond 20 weeks of gestation leading to higher birth weight even in the preeclampsia group. Mean values of age, BMI, serum creatinine, and platelets are comparable to the present study.

Mean CA-125 values were higher in the preeclampsia group in all studies including the present study thus supporting the hypothesis that increasing severity of the disease leads to increased inflammatory response causing increased CA-125 release.

\section{Conclusion}

The present study aimed at measuring CA-125 levels in preeclampsia and gestational hypertension and in normal pregnancy. It was found to be significantly elevated in preeclampsia. Cancer antigen 125 value had a positive correlation with other clinical parameters such as systolic and diastolic blood pressure, uric acid, and other outcomes of preeclampsia such as fetal growth restriction and oligohydramnios, thus indicating that it is a marker of increasing severity of the disease. Cancer antigen 125 is a biochemical marker which can be used in the screening of preeclampsia. Further studies are needed for the evaluation of raising serial CA-125 levels as a marker of progression of the disease.

\section{Clinical Significance}

Hypertensive disorders of pregnancy are one of the most common causes of death due to pregnancy. Cancer antigen 125 was proposed to be elevated in patients with hypertension due to chronic inflammation. Hence, CA-125 levels if used as screening tool can estimate the severity of the disease and help clinicians in identifying women who are at risk for further complications and act accordingly.

\section{Acknowledgments}

Authors would like to extend their thanks to Department of Biochemistry, Kasturba Medical College, Mangaluru, for carrying out ELISA tests and to the Head of the Department, Obstetrics and Gynaecology, for giving the authors the opportunity to carry out this study. It is a self-aided study.

\section{References}

1. Arulkumaran N, Lightstone L. Severe pre-eclampsia and hypertensive crises. Best Pract Res Clin Obstet Gynaecol 2013;27(6):877-884. DOI: 10.1016/j.bpobgyn.2013.07.003.

2. Martin JNJ, Owens MY, et al. Standardized Mississippi Protocol treatment of 190 patients with HELLP syndrome: slowing disease progression and preventing new major maternal morbidity. Hypertens pregnancy 2012;31(1):79-90. DOI: 10.3109/10641955.2010.525277.

3. Suh KS, Park SW, et al. Ovarian cancer biomarkers for molecular biosensors and translational medicine. Expert Rev Mol Diagn 2010;10(8):1069-1083. DOI: 10.1586/erm.10.87.

4. Malatyalioglu E, Ozer S, et al. CA-125 levels in ruptured and unruptured tubal ectopic pregnancies. J Obstet Gynaecol Res 2006;32(4):422-427. DOI: 10.1111/j.1447-0756.2006.00424.x.

5. Lindheimer MD, Taler SJ, et al. Hypertension in pregnancy. J Am Soc Hypertens 2010;4(2):68-78. DOI: 10.1016/j.jash.2010.03.002.

6. Cebesoy FB, Balat O, et al. CA-125 and CRP are elevated in preeclampsia. Hypertens Pregnancy 2009;28(2):201-211. DOI: 10.1080/10641950802601187.

7. Bhattacharya DA, Saha R. Serum concentrations of CA-125 in normal and Preeclamptic pregnancies. IOSR J Pharm 2014;8:14-17. DOI: 10.9790/3013-0408014017.

8. Karaman E, Karaman Y, et al. Maternal serum CA-125 level is elevated in severe preeclampsia. Pregnancy Hypertens 2014;4(1):29-33. DOI: 10.1016/j.preghy.2013.08.003.

9. Hassan MA, Hameed BH, et al. The Association of Serum Cancer Antigen 125 and $C$ - reactive protein Level with The Severity of Preeclampsia. Karbala J Med 2012;5(1):1322-1327.

10. Ozat M, Kanat-Pektas M, et al. Serum concentrations of CA-125 in normal and preeclamptic pregnancies. Arch Gynecol Obstet 2011;284(3):607-612. DOI: 10.1007/s00404-010-1736-2. 